\title{
Stratégie de survie et culture de jeunes dans les marchés urbains de Dakar : cas des adolescentes travailleuses (Sénégal).
}

\author{
Rosalie A Diop \\ SociologueAssistante/Chercheure à l'IPDSR/UCAD \\ Email : rosaliedk2005@yahoo.fr \\ Tél: $8254001 / 5247391$
}

\begin{abstract}
Résumé
Cet article porte sur la vie quotidienne des adolescentes travailleuses dans les marchés urbains et péri-urbains de Dakar, capitale du Sénégal. Elle vise à approfondir la compréhension et la connaissance sur les stratégies de survie et la socialisation des jeunes dans les rues des villes sénégalaises et sur les logiques de celles-ci dans un contexte social spécifique. Le travail des adolescentes recouvre des activités hétérogènes conçues sous l'angle des stratégies de survie Plusieurs facteurs socio-démographiques et éléments sociologiques expliquent l'insertion précoce des adolescentes dans les activités de production et dans le petit-commerce, en particulier. Les raisons, les motivations et les intérêts des adolescentes d'exercer ce travail sont pluriels mais une logique importante et rationnelle apparaît et réside dans leur contribution à la survie familiale.. Dans ce cadre, il représente pour elles une quête positive de sens construite autour de la recherche d'une survie instrumentale, de la satisfaction des besoins personnels et de la conquête d'un nouvel espace de réalisation. Les stratégies développées participent à la construction progressive de leur identité et permettent une reconnaissance sociale. Ces activités leur permettent aussi de se construire en tant qu'actrices et d'augmenter leur zone d'autonomie et de liberté. Cela exerce une influence positive sur la transformation de leur statut et sur la place quelles occupent dans la société.
\end{abstract}

Descripteurs: Adolescence, Jeunesse, Socialisation, Pauvreté, Exclusion, Secteur informel urbain, Stratégie de survie, Activités Carrière, Marché, Genre 


\section{Introduction}

Cet article situe le travail des adolescentes non dans une approche de victimisation, ni de protectionnisme, mais dans une perspective alternative. La problématique des adolescents et des jeunes en Afrique est assez complexe et interpelle tous les acteurs sociaux : adolescents eux-mêmes, chercheurs, bailleurs, intervenants. Les questions sociales qui l'entourent sont vastes, diversifiées et d'actualité et peuvent être regroupées sous plusieurs thèmes, notamment les questions de pauvreté/exclusion, de l'éducation/formation, de l'emploi, des nouvelles technologies de l'information et de la communication ; les adolescents face aux risques, c'est-à-dire les questions prioritaires de la for services aux soins, de toxicomanie, de la délinquance, des conflits intergénérationnels, etc.

Nous nous proposons dans article d'examiner comment elle se pose dans les communautés d'adolescentes défavorisées, où se développent des alternatives de survie et de carrières.

La carrière des adolescentes travailleuses est progressive et la manière dont nous la présentons mérite certainement quelques validations car il reste de nombreuses hypothèses à vérifier. La formalisation de la carrière de petite-vendeuse en tant que construction d'idéal-type permettra de reconstituer les différentes phases et de comprendre certains aspects de la trajectoire, de l'identité en plus de donner un contenu aux logiques du travail des adolescentes. Rappelons, à ce niveau, que le travail des adolescentes constitue des activités de survie très variées quant à leurs ambitions, leurs valeurs et leurs ressources.

Différents éléments participent à la construction de la carrière des adolescentes travailleuses. Sous cet angle, la carrière des adolescentes travailleuses pourrait certainement être lue et étudiée de diverses manières compte tenu des activités, du cadre, etc. Nous pouvons citer l'expérience, le temps, les réseaux de sociabilité, la socialisation, le genre, l'environnement social, l'espace de travail, l'identité, etc.

\section{Définition des Concepts}

\section{L'adolescent un concept en construction}

Notons d'emblée que les recherches africaines en sciences sociales sur la problématique de l'enfance, de l'adolescence et/ou de la jeunesse en difficulté (grossesse d'adolescentes, enfants et jeunes de la rue, enfants au travail, etc.) ne sont apparues que depuis une dizaine d'années. Deux raisons principales sont évoquées pour expliquer cette situation.

Selon certains auteurs (Coquery-Vidrovitch, Goerg, Topor, 1991), la première raison est 
d'ordre démographique. Elle est due au retard de « l'explosion démographique » dans les pays africains. La deuxième raison est liée aux facteurs sociologiques des « conceptions africaines de l'âge, du savoir et du pouvoir ${ }^{1}{ }^{\star}$.

L'analyse des différents écrits montre qu'il semble exister un certain consensus quant à la délimitation du concept de jeunesse. Celle-ci se diviserait en trois périodes : l'enfance proprement dite, l'adolescence et la jeunesse. Toutefois, les tranches d'âge associées à chaque période varient énormément. À cette fin, la construction de ces concepts constitue un travail scientifique difficile même si son utilisation semble évidente. L'analyse des études existantes sur la composition empirique de ces concepts montre une grande variation quant à son utilisation, ce qui soulève de multiples polémiques et controverses entre les intervenants et les différents spécialistes en sciences sociales.

Le concept d'adolescence (qui nous intéresse dans cette communication) est apparu récemment dans les recherches en sciences sociales (entre la fin du XIXe siècle et le début du XXe). Cependant, il a surtout été abordé sous l'angle des « déterminants du comportement sexuel précoce avant ou au sein des mariages, soit ceux de la nuptialité ou encore ceux des grossesses et maternités précoces » (Evina, 1998). Malgré certaines divergences, sa définition transitionnelle en tant que stade débutant à la fin de l'enfance et se terminant au moment de l'âge adulte marquée par la puberté semble faire l'unanimité.

En résumé, dans la littérature consultée, nous sommes confrontée à une grande diversité et à une grande élasticité des classes d'âge quand on parle de l'enfance (moins de 10 ans), de l'adolescence ou de jeunesse (10 -19 ans, 15-19 ans, 15-24 ans : CoqueryVidrovitch, 1992 ; Delaunay, 1996). Dans la plupart des études concernant ces catégories, chacune utilise des classes d'âges différentes. Aussi, cette classification dépend toujours de critères statistiques très relatives et subjectives. Les transformations dans les sociétés africaines et leur rapprochement des sociétés occidentales industrielles en regard de certains paramètres nous autorisent à nous inspirer de certaines thèses concernant la jeunesse.

En effet, il est aujourd'hui largement admis que l'adolescence et la jeunesse sont des produits spécifiques de la modernité (Diop,1995). ${ }^{2}$ En effet, ces deux concepts sont apparus au début du siècle avec le développement du phénomène urbain, de la scolarisation et des nombreuses autres transformations qui ont eu lieu dans les sociétés modernes.

En nous inspirant de Galland, nous pouvons dire que l'adolescent à l'instar de la " jeunesse est le résultat d'une construction socio-historique constamment en redéfinition dans la société moderne, elle constitue la période de la vie qui s’allonge » (1993). Plus

1 En Afrique, la société est fondée en grande partie sur la séniorité. La détermination de l'âge est ainsi liée à l'expérience et aux initiations qui peuvent surgir tôt ou tard dans la vie de l'individu.

2 Les études ethnologiques nous ont fait connaître de nombreuses sociétés traditionnelles où il n'y a pas d'adolescence. Dans ces sociétés, les garçons et les filles, passent directement de l'enfance à l'âge adulte avec tous les droits et les devoirs des adultes. Cette transition ne peut évidemment être appelée adolescence au sens où on entend maintenant ce mot c'est-à-dire comme une période caractérisée par un statut social différent à la fois de celui de l'enfant et de celui de l'adulte. 
encore « l'adolescence serait donc une époque où le jeune perd son statut d'enfant sans acquérir les privilèges liés au statut d'adulte» (Côté, 1991 : 147).

Dans cet article il s'agit d'adolescents généralement âgés de moins de 20 ans, confrontés très tôt au monde de la production de biens et services (économiques) qui a retenu notre attention. Dans son passage de l'enfance à l'âge adulte, l'adolescent vit certaines angoisses psychologiques que les spécialistes qualifient de crise d'adolescence. Cette crise se trouve aujourd'hui amplifiée par les dures conditions de vie, par un avenir incertain (abandon et ou échec scolaire), par le manque de moyens pour poursuivre des études ou faire une formation, par la pauvreté sous toutes ses dimensions. Cette crise d'adolescence est beaucoup plus manifeste dans les villes où d'autres réalités font leur apparition.

\section{Le concept de travail des enfants comme stratégie de survie}

Quand on développe la problématique « des enfants travailleurs » comme stratégies de survie, on perçoit très vite une sorte d'aporie dans la définition des concepts. En effet, les recherches sur le travail des enfants et des jeunes analysées comme une stratégie de survie en sont encore à leurs débuts. En conséquence, le sens donné par les chercheurs illustre plusieurs controverses. Elles sont liées à des problèmes d'ordre conceptuel et épistémologique.

Les notions " enfants travailleurs " sont vastes, complexes et sont sources de controverses surtout quand elles s'appliquent à des enfants et encore plus à des filles. Elles présentent des problèmes d'ordre conceptuel et empirique. Ce qui nous conduit à établir une distinction opératoire entre le travail des enfants dans « l'activité domestique » (child work) qui correspond aux tâches ménagères assumées par l'enfant fille ou garçon pour le fonctionnement de l'unité familiale dans son processus de socialisation (Erny, 1981 ; Mbodj, 1993) ou dans le cas d'un apprentissage professionnel (Enda Tiers-Monde, 1995) et le travail des enfants (child labour) orienté par la recherche d'un gain monétaire 3 . Cette forme de travail renvoie à une activité productive génératrice de revenus à plein temps. Elle implique une part plus ou moins grande d'abus et d'exploitation.

En partant de ces différents points de vue y compris celui des adolescentes et de nos observations, nous cherchons à déconstruire la notion d'« enfants travailleurs » afin de mettre en évidence les comportements quotidiens et certaines caractéristiques des activités (qui auront trait aux motivations que nous verrons plus loin) des enfants et surtout des adolescentes dans les marchés et parfois dans les rues. Nous considérons le travail des adolescentes comme une activité économique concourant à la production de biens et de services. Cette forme de travail relève de stratégies de survie les plus couramment adoptées par une population juvénile, pauvre et moyennement défavorisée

3 L'apprentissage professionnel apparait comme un processus par lequel on acquiert un ensemble de connaissances pratiques, un savoir-faire, un savoir être et un savoir-vivre. 
en milieu urbain pour résister à la crise, à la pauvreté ou à l'exclusion. En dépit des nombreuses législations nationales, régionales et internationales, le travail constitue, aujourd'hui plus que jamais, le principal moyen de «survie » ou de « débrouillardise » de milliers d'adolescentes et d'adolescents défavorisés au Sénégal. Ces stratégies de survie constituent des pratiques visant à atteindre un but, une fin, elles se rattachent à un ensemble d'activités de production de biens et de services. Cette définition ou tentative de définition se veut pragmatique et opérationnelle.

\section{Les etaPes de la carrière des adolescentes}

Dans cette section, nous reconstituons la carrière de petite-vendeuse en quatre phases : 1) l'entrée qui correspond à la motivation ; 2-3) deux phases du développement : la fragilisation et l'accommodation; 4) la sortie qui correspond aux projets et aux rêves que nous identifions comme étant l'avenir.

\section{La motivation}

Qu'est-ce qui peut bien motiver des adolescentes à travailler dans les marchés ? En tant qu'actrices, ces adolescentes fournissent des explications sur les relations qu'elles entretiennent avec leur activité.

Il faut dire qu'au-delà de la motivation originelle liée à la subsistance familiale, les adolescentes évoquent plusieurs motifs à la fois utilitaires, fonctionnels, identitaires, ludiques ou socialisants, etc. Ces composantes rejoignent les recherches effectuées sur les enfants de la rue ou sur les enfants travailleurs dans la rue en Amérique Latine ${ }^{4}$. Selon ces recherches, ces fonctions sont souvent interreliées et parfois difficiles à départager. Du point de vue des adolescentes, la composante utilitaire est la plus motivante et elle est de loin le motif fondamental. Elle s'explique par les gains obtenus et par leur utilisation. Cependant, nous remarquons que, pour certaines adolescentes, la lutte contre l'oisiveté constitue une motivation aussi importante que la dimension économique. Il ne faut pas oublier que la majorité des adolescentes ne vont plus à l'école et quelles sont sans travail formel.

Les modalités d'entrée sont liées à des situations globales de précarité causées par les conditions de vie difficiles. L'entrée des adolescentes est définie ici comme le processus selon lequel l'adolescente évolue dans un secteur d'activité représenté par le micro-commerce afin d'en apprendre les rudiments. Il est évident que la pauvreté, les crises socio-économiques liées aux conditions macro-économiques expliquent la venue des adolescentes dans les marchés. Cependant, de manière objective, la situation des adolescentes est dépendante des revenus de la famille. Or, nous l'avons vu, les revenus familiaux sont très précaires et irréguliers, sinon absents. Pour la majorité des

4 Voir les travaux de Lucchini 1993, 1996 et Invernizzi, 2001. 
adolescentes, le père ou le frère ne travaille pas du fait de la retraite, d'un licenciement, de la fermeture de l'usine, du chômage des jeunes. Ces différentes situations entraînent des pertes ou des absences importantes de revenus. L'absence de ressources financières et de ressources familiales devient, pour toutes les filles, la raison première de leur mise au travail et de leur arrivée dans le petit-commerce, sur recommandation des parents. Les expressions : « nous n'avons pas les moyens »; « nous n'avons pas d'argent »; « nous n'avons pas de ressources »; " personne ne nous vient en aide »; " mon père ne travaille pas »; « mon père ne travaille plus »; « ma mère n'a personne sur qui compter »; « mon père est mort et ma mère est seule » etc., illustrent la situation de plusieurs adolescentes qui nont aucune prise sur leur condition de vie. Les raisons sont simples et elles se résument à l'assistance à la mère dans le commerce et à une aide pécuniaire à la famille. Car, comme le témoigne le discours récurrent des adolescentes « sans argent on n'est rien, on ne fait pas partie des gens valorisés de ce monde d'aujourd'hui ».

Donc, la première raison d'entrée dans la carrière est déterminée par un souci de résoudre le problème urgent de subsistance. Le travail des adolescentes, dans ses conditions, s'explique à partir du lien avec la famille; elles associent le travail à un devoir, à un contrat moral, à une " dette sociale » envers la famille. Ces expressions illustrent cette condition morale : «il est de mon devoir d'assister ma mère et de connaitre les ficelles du métier et, pourquoi pas plus tard, prendre la relève car, tôt ou tard, elle sera obligée de quitter le marché à cause de son âge »; « je dois soutenir ma mère pour les nombreuses dépenses de la maison ".

Un autre aspect lié au travail des adolescentes est la valeur de la participation de tous les membres de la famille permettant une amélioration des conditions socioéconomiques et non pas seulement pour la subsistance. Beaucoup d'adolescentes contribuent ainsi à financer la scolarité de leurs frères ou de leurs sœurs plus jeunes, certaines règlent les factures d'électricité, d'eau ou contribuent au paiement du loyer.

L'autre fonction utilitaire du travail est qu'il représente une source de satisfaction des besoins que les parents ne peuvent pas assumer (habillement, produits de beauté, chaussures ou argent pour les cérémonies...). La satisfaction des besoins matériels personnels constitue de nouvelles donnes pour les adolescentes en situation de pauvreté qui doivent se prendre en charge. Cette dimension est apparue dans tous les récits des adolescentes « je dois régler mes problèmes » ou « je dois subvenir à mes besoins ».

Les adolescentes travailleuses sont des actrices de leur propre survie et de la vie de leur famille. Cet aspect du travail n'est pas analysé. La plupart des analyses sur le travail des adolescentes le réduit à sa dimension économique à un homo oeconomicus. Par ailleurs, les rationalités autres que productives sont minimisées. Or, les motifs ne reposent pas uniquement sur la rationalisation économique ou instrumentale. D'autres rationalités (relationnelle, symbolique, socialisante) sont mises en évidence par les adolescentes interrogées. Ces rationalités informent sur l'identité, sur la personnalité et sur l'environnement social des adolescentes. 


\section{La fragilisation}

La fragilisation apparaît au début de l'entrée des adolescentes dans les activités au marché. Tout au long de la communication nous avons raisonné en termes de processus pour apprécier les situations de fragilisation des adolescentes. Nous n'allons pas y revenir, relevons simplement que notre construction sur les activités de survie s'appuie sur des logiques instrumentales concrètes. Les adolescentes sont arrivées au marché par obligation ou contrainte économique, contrainte de la survie familiale ou de la survie individuelle. Les conditions de l'entrée précoce des adolescentes en activité dans le petitcommerce au marché sont différentes d'une adolescente à une autre. Malgré cela, elles présentent plusieurs similitudes. La fragilisation peut être liée à un début de socialisation par l'activité, l'intégration et l'adaptation. Elle peut être différente d'une adolescente à une autre compte tenu des raisons d'entrée dans le commerce et dans le marché. Pour certaines adolescentes dont l'entrée n'est pas guidée, l'insertion dans l'espace-marché et dans les activités est parfois difficile.

\section{L'accommodation}

L'accommodation correspond à la période d'engagement et d'adaptation de l'adolescente dans son milieu et dans ses activités. Avec la durée, l'expérience acquise, les modalités de la vie quotidienne au marché, la routine s'installe progressivement. Le travail au marché devient un habitus pour reprendre Boudieu. Les adolescentes ont pu se familiariser, s'habituer et s'adapter à l'espace marché qu'elles s'approprient. Aussi, elles déploient des habilités, des savoir-faire et des pratiques concrètes qui leur permettent de s'engager et de développer des interactions avec les différents acteurs du marché. À travers le temps, elles développent des stratégies, des tactiques, des rituels qui sont à la fois matériels, individuels et symboliques. Au fur et à mesure quelles vieillissent, elles acquièrent de l'expérience, voire de l'assurance. Elles y prennent goût et décident, à un moment donné, de se mettre à leur propre compte. La longue fréquentation du marché, l'insertion dans les réseaux de sociabilité, la connaissance des ressources disponibles permettent aux adolescentes de maitriser leur existence quotidienne. En surmontant et en acceptant leur condition et leur destin, les adolescentes parviennent à s'approprier le milieu et à prendre contact avec les pairs et les autres acteurs avec qui il sera possible d'échanger des ressources (affectives, matérielles), des fournisseurs et des clients.

La période d'accommodation passée, les adolescentes commencent à nourrir des projets et à saisir les différentes opportunités présentes au marché. Cette accommodation et ces stratégies seront construites différemment. Les problèmes rencontrés, les souffrances, la fatigue, le désespoir se font sentir car certaines filles font l'objet de harcèlement sexuel, d'exploitation, d'abus de toutes sortes en plus les revenus sont très précaires. Le contact 
avec l'argent augmente pour certaines les besoins et la dépendance, qui parfois les orientent dans des pratiques déviantes : le cas de Rose est éclairant. Nous le rappelons, elle affirme avoir plusieurs fars (petits amis) à qui elle demande de l'argent pour régler des besoins ponctuels. Des pratiques parfois orientent parfois les filles vers la prostitution formelle.

\section{Les perspectives d'avenir}

Les sorties ou les perspectives prennent des formes différentes selon le genre, l'âge, l'activité et la représentation que l'adolescente se fait de son travail et du milieu. Contrairement à la carrière des enfants et des jeunes en rupture qui ne dure pas longtemps, la carrière liée aux activités de petit-commerce dans les rues et surtout dans les marchés peut durer longtemps et souvent très longtemps. Aussi, nous parlerons plutôt de perspectives pour les adolescentes travailleuses. Plusieurs possibilités peuvent être envisagées quant à l'avenir. Il peut dépendre de l'organisation familiale : amélioration des conditions de vie ou du revenu familial. Dans ce cas, les parents ou l'adolescente peuvent envisager le retour aux études de l'adolescente aux études notamment à une formation si elle est déjà scolarisée. Ces cas sont assez rares. Certains parents, même s'ils manifestent le souhait de voir leurs enfants retourner à l'école, ne parviennent pas à trouver les ressources nécessaires pour la survie concrète (manger, se loger). À ce propos, nous remarquons que le discours des ONG et des institutions met l'accent sur les liens entre la scolarité, le travail des enfants et leur avenir. On oublie que dans un contexte de précarisation et dans un processus d'exclusion, l'espoir d'une profession ou d'un avenir par une progression socio-économique ou par la scolarisation prolongée peut constituer une utopie, quelque chose de très éloigné de la réalité quotidienne. La scolarisation ne constitue pas une référence positive pour des individus qui luttent pour leur survie et encore moins pour des adolescentes qui sont exclues des circuits formels d'éducation. Nous estimons que la scolarisation des filles, et des filles pauvres en particulier, ne se fera pas si des modifications ne sont pas apportées pour améliorer les revenus des parents et de la mère, notamment.

L'analyse des possibilités de changement de carrière dépend aussi des rapports de genre. Pour les filles rencontrées, le besoin d'améliorer ou de changer leur condition de vie par le statut et par l'identité dépend de leur insertion sociale. Ces possibilités sont individuelles et dépendent directement de l'adolescente. Des expressions locales dénotent de leur volonté d'améliorer ou de changer leur condition de vie et même celle de leur famille : am diekeur bou bakh (avoir un bon mari) ou tuukki (voyager), faire une formation ou faire de l'import-export. Les adolescentes sont conscientes que la scolarisation ou la formation professionnelle ne garantit pas l'obtention d'un emploi formel ni de revenus importants. Aussi, leurs propos sur la formation cadrent avec leurs besoins de carrières de vendeuses : formation en informatique, en comptabilité, en gestion pour mieux tenir 
leur commerce. En somme, elles désirent améliorer leurs conditions de travail. Dans le cadre de la lutte contre la pauvreté, notamment dans les programmes d'amélioration des conditions de vie et de protection des enfants et adolescentes, cette dimension doit être prise en compte pour la réalisation de leur projet.

Pour presque toutes les adolescentes, la sortie du marché ou l'accession à un autre travail et la reconnaissance sociale adviennent par le mariage. Dans ce cadre, elles sont prises en charge, ce qui leur permet de réaliser certains de leurs projets dont le fait d'avoir une maison et/ou paradoxalement, continuer leur commerce mais à une échelle beaucoup plus grande.

Un fait nouvellement engendré par la crise et la pauvreté est que les femmes et les filles ne semblent plus vouloir rester dans les maisons, ce qui contraste avec les femmes plus âgées et la vision stéréotypée que l’on se fait du statut et de la place de la femme car, traditionnellement, la femme sénégalaise était censée rester dans la sphère privée. De plus en plus de femmes et d'adolescentes préfèrent le contact direct avec le monde extérieur et être informées de ce qui s'y passe. Il se dégage du discours des adolescentes, de manière récurrente, les expressions locales Dem thi kaw, fi yorou ka, dem été dji qui signifient " aller à l'étranger », " il n'y a rien dans ce pays », " encore profiter de l'été ». Voyager ou partir fait partie des nombreuses stratégies de survie adoptées par les jeunes et, aujourd'hui, par les femmes. C'est un rêve, voire une obsession pour presque tous les jeunes des différentes couches sociales au Sénégal. Par malheur, certaines sont prêtes à n'importe quoi pour quitter le pays en passant par des mariages d'intérêt avec des étrangers, par exemple ${ }^{5}$. Elles partent pour des périodes déterminées, généralement l'été, pour une durée de quatre à six mois. Il va sans dire que cette nouvelle forme de migration des jeunes filles et des adolescentes comporte beaucoup de risques du fait de leur jeunesse et de leur inexpérience.

\section{La carrière de petite vendeuse : genre et représentations}

Les rapports entre le genre et la trajectoire des mères influent largement la carrière des enfants travailleurs et notamment celle des petites-vendeuses. La représentation que les adolescentes ont de leurs activités résulte d'éléments subjectifs qui leur sont extérieurs et qui ont un caractère plus ou moins contraignant.

Pour comprendre la carrière de petite-vendeuse, il est indispensable de considérer les normes de socialisation selon le genre. Nous avons vu, à travers nos recherches, que le statut et les rôles sont déterminés par une approche déterministe de la socialisation différentielle qui met l'accent sur les stéréotypes, les interdits et les stigmates. En ce

5 La société tolère le mariage avec les étrangers quoique certaines formes du mariage soient en contradiction avec les valeurs sociétales et religieuses. Certains parents, à cause des contraintes et des pressions économiques, acceptent ces mariages quills essayent de justifier et de légitimer au sein de la société. 
sens, la socialisation a contribué de manière importante à la construction des identités masculines et féminines et à leurs représentations. Nous comprenons pourquoi, aujourd'hui, malgré l'évolution et les changements sociaux, la perception que les individus ont à l'égard du travail des adolescentes n'a pas beaucoup évoluée. Dans cette perspective, nous estimons qu'une approche interactionniste et inter-culturelle qui prendrait en considération les schèmes socioculturels et subjectifs permettrait de mieux éclairer le choix des activités des adolescentes dans les marchés. Cependant, comme le mentionne Invernizzi (2001), la socialisation de genre n'est pas univoque. En effet, elle se construit parfois sur des attentes contradictoires et entraîne des changements qui vont à l'encontre des rôles de genre prescrits. Dans ce cas, ces contradictions peuvent être source de souffrances, d'inégalités car ne correspondant pas aux stéréotypes et représentations féminines et masculines; d'où le caractère paradoxal du travail des adolescentes dans les marchés et même dans les rues hors du cadre normatif de la sphère privée. Il s'agit de contradictions existantes entre des logiques subjectives de socialisation et les logiques de survie dans un contexte de pauvreté.

\section{La représentation de l'environnement de travail : l'espace-marché}

Les liens que les adolescentes entretiennent avec le travail, le marché ou leur environnement dépendent d'un ensemble d'éléments différents. La conception du lieu de travail est différente selon les types d'activité et aussi selon le genre.

Nous avons posé comme hypothèse que les marchés urbains et les marchés de rue constituent de nouveaux espaces investis par des populations défavorisées dont les adolescentes en quête de survie et d'identité. Nous avons particularisé l'espace-marché car, jusqu'à présent, la plupart des études sur le travail des enfants et des adolescents se sont limitées ou concentrées sur la rue. Or, le marché constitue un cadre spécifique fermé et totalement différent de la rue. Dans cette perspective, le marché offre plus de protection. Dans le monde urbain sénégalais, la rue est perçue comme un espace arbitraire, essentiellement masculin et donc, à travers les modèles traditionnels, excluant une occupation de la rue par des filles. Pour ces adolescentes, l'espace-marché est connu et le plus souvent maîtrisé, contrairement à l'espace-rue. Cependant, les marchés sont aussi différents que les activités qui s'y pratiquent. La question est de savoir si l'insertion des adolescentes dans les marchés constitue de nouvelles formes de sociabilité ou, au contraire, si elles apparaissent comme des conditions de rupture ?

L'insertion des adolescentes dans les marchés urbains peut apparaître comme une rupture volontaire ou involontaire des espaces de socialisation traditionnels. Cependant, nous pensons que ce passage dans le marché, loin dêttre une rupture, constitue une évolution ou un glissement de la socialisation hors des normes conventionnelles. Cette entrée dans le marché représente pour certaines adolescentes une distanciation progressive, un éloignement du milieu familial. Dans ce contexte, les filles ont tendance 
à adopter des valeurs différentes.

Il existe effectivement une différence fondamentale entre les jeunes filles qui exercent des activités de survie dans les marchés (comme nous venons de le voir) et les adolescentes en rupture partielle ou totale avec la cellule familiale qui, en raison de conditions de vie difficiles, de pauvreté, de victimisation physique, d'abus sexuel, de violence familiale, etc., se sont écartées des voies traditionnelles de la socialisation et ont adopté la rue comme nouvel espace de résidence ou de vie. Pour ces dernières, la rue constitue "l'ultime espace » auquel elles ont recours pour vivre de manière temporaire ou permanente et souvent pour réussir une quête d'autonomie qu'elles nont pu réaliser ailleurs (Bellot,2001). Ce que Lucchini (1996) appelle la dialectique entre la socialisation familiale et la socialisation de la rue, théorie ancienne de la sociologie classique sur la délinquance juvénile américaine. En conséquence, certaines activités qu’elles exercent dans la rue (prostitution la plupart du temps, vol à la tablette, recel de drogues ou autres) leur permettent tout bonnement de vivre dans la rue et de mieux s'adapter à la culture de la rue qui appelle à d'autres formes de socialisation et de sociabilité telles l'appartenance à des groupes ou sous-groupes, à des bandes, à des réseaux ou à des dyades.

Les adolescentes que nous avons rencontrées ne sont pas en rupture avec le milieu familial. Au contraire, pour beaucoup d'entre elles, la cellule familiale joue un rôle déterminant dans leur quête de survie et d'autonomie identitaire. Les marchés urbains représentent des espaces alternatifs permettant de réaliser des activités de production de façon temporaire ou permanente et souvent pour réussir une quête « d'autonomie, de liberté et de dignité » qu'elles ne peuvent faire autrement. Ce choix est exprimé de manière récurrente par la plupart des adolescentes qui, durant leur trajectoire professionnelle, ont travaillé comme domestiques de maison. Le marché, dans ce cadre, signifie liberté, nouvelle autonomie et espace de réalisation des besoins. Cependant, la réalisation de ces besoins, ne se fait que dans le cadre d'un système de réseautage familial ou relationnel à travers des associations. Les adolescentes sont en quête, dans leurs interactions, d'une reconnaissance personnelle positive et d'une valorisation de soi. Partant de ce point de vue, nous pouvons affirmer que le marché participe à la construction des adolescentes qui, grâce au contact avec d'autres personnes et en particulier avec le monde des adultes, se structurent. Cette construction de soi passe à travers la quête d'une autonomie économique et le développement des stratégies pour la survie. La connaissance du milieu et la présence des parents minimisent les risques d'agression et de victimisation qui sont présents dans les marchés. Comme Young $(2003)^{6}$ l'a fait pour les enfants de la rue, nous avons mis l'accent sur l'ingéniosité et les

6 "This paper examines the 'place' of street children in Kampala, Uganda highlighting their ingenious and resourceful use of the urban environment. Considered 'out of place' in urban public space, street children create their own niches in the marginal spaces of the city. By looking at untouchable spaces, underground spaces and rooftop spaces this paper shows how they develop their own place identities. For survival, street children react to their exclusion by resisting this out of place image and encroaching into crowded spaces or by dominating the street under the cover of darkness. However, in certain city spaces, street children are also legitimised and 
ressources des adolescentes dans un environnement urbain. Nous avons aussi, comme cette auteure, observé que les adolescentes créent leurs propres « niches » ou leurs propres espaces. Ces espaces marginaux sont investis par les adolescentes et leur permettent d'y développer leur propre espace identitaire. Pour survivre et réagir à leur propre exclusion, des adolescentes ont résisté à cette mise à la marge en s'appropriant, de l'intérieur, des espaces publics dont l'espace-marché.

En conclusion, nous pouvons dire que cette manière originale permet de légitimer leur présence dans cette sphère d'activités ; cela transforme progressivement ces activités marginales, en activités acceptables, acceptées, voire normalisées. Cependant, si le marché comme espace apporte des réponses positives aux vécus quotidiens des adolescentes, il constitue encore, selon celles-ci, un lieu de désocialisation et de resocialisation qui est en contradiction avec les valeurs traditionnelles. D'ailleurs, lorsqu'au Sénégal, on entend dire "Kii khale marsé " (c'est " un enfant de marché »), cela veut dire beaucoup de choses et, entre autres, « c'est un enfant dévergondé, qui n'a pas froid aux yeux ou qui n'a pas d'éducation ». Ce stéréotype est renforcé par la conception sénégalaise de la place et du statut de la femme. Dans l'imaginaire collectif et dans l'imaginaire des hommes notamment, les filles doivent rester à l'intérieur du foyer. Par conséquent, les filles qui ne correspondent pas à cette image sont stigmatisées comme de mauvaises filles, des dévergondées ou des assimilées. Aussi, pour certaines filles ambulantes qui n’ont pas de place fixe de vente, le marché peut être une source de dévalorisation à cause d'une nonreconnaissance statutaire.

\section{Conclusion}

L'analyse des motifs de l'entrée des adolescentes dans des activités de production met en évidence la dimension multi-fonctionnelle du travail des adolescentes et la place primordiale accordée à la survie familiale. Le discours des adolescentes démontre que leurs activités considérées comme des stratégies de survie vont au-delà des critères objectifs, statistiques et économiques universellement reconnus. Elles englobent d'autres dimensions subjectives, culturelles, sociales, contextuelles et conjoncturelles. Cependant, la survie constitue l'enjeu fondamental et revêt toutes les caractéristiques de la rationalité du modèle économique de type instrumental. Devant les difficultés économiques, les efforts de toute la famille pour participer à la survie familiale sont obligatoires et appréciables. Tous les membres de la famille s'adonnent à des tâches productives, différenciées suivant l'âge et le sexe. Les changements dans le mode de production ne

accepted by the social street if they conform to the desired behaviours of that space. The place of street children in Kampala is one which is contested, resulting in a multiplicity of street children's niches being created which vary both spatially and temporally." Lorraine Young, 2003. "The 'place' of street children in Kampala, Uganda: marginalisation, resistance and acceptance in the urban environment». Environment and Planning D : Society and Space, p. 2. 
transforment pas immédiatement, ni automatiquement le travail des adolescentes. Ils ne modifient pas directement les stratégies de reproduction et l'organisation de la famille qui, elle, exerce une influence sur les activités productives des adolescentes. L'histoire du travail des adolescentes doit être saisie à la lumière des changements économiques, démographiques mais aussi culturels.

Amplement déterminée par le parcours social et scolaire antérieur, la mise au travail des adolescentes dans les marchés urbains n'est cependant pas vécue seulement sous un mode négatif. Pour ces adolescentes, il apparaît comme un choix raisonné et rationnel, comme le meilleur choix. Tout en leur permettant de demeurer dans leur milieu social et leur groupe d'amis, ce choix leur permettra d'acquérir rapidement une indépendance financière, une autonomie identitaire à l'égard de leurs parents et d'accéder au statut d'adulte sans être happées par des problématiques de prostitution, de drogues ou autres. Le fait que les adolescentes aient appris à travailler, à gagner de l'argent et à participer à la gestion familiale les valorise et elles se sentent respectées au sein de la famille et du quartier. Certaines se sentent plus libres et plus indépendantes et les stratégies de survie les survalorisent. Pour d'autres, ces stratégies de survie participent à leur formation et à leur apprentissage social. Cependant, il ne faut pas faire l'impasse non plus sur le fait que certaines stratégies de survie augmentent les problèmes de certaines adolescentes déjà fragilisées par la crise d'adolescence et leurs conditions de vie difficiles. 


\section{Références}

Aduayi, Rosalie-Diop. 1995. « Crise de la famille : enfants et jeunes en rupture à SaintLouis. Quelles stratégies de réinsertion familiale ? ». Mémoire de maîtrise en sociologie, Saint-Louis (Sénégal), Université de Gaston Berger à Saint-Louis, $107 \mathrm{p}$.

Aguessy, D. 1968. « La femme Dakaroise commerçante au détail sur le marché ». Dakar en devenir, sous la dir. de M Sankalé, Louis-Vincent Thomas et Pierre Fougeyrollas, p. 396-419. IFAN : Présence Africaine.

Amina, Mama. 1997. Études par les femmes et études sur les femmes en Afrique durant les années 1990, Dakar : Codesria, $131 \mathrm{p}$.

Antoine, Philippe, Abdou-Salam Fall, Agnés Adjamagbo et al. 2002. Crise, passage à l'âge adulte et devenir de la famille dans les classes moyennes et pauvres à Dakar. Dakar : IFAN-CODESRIA-RD.

Bellot, Céline. 2001. «Le monde social de la rue : expériences des jeunes et pratiques d'intervention à Montréal ». Thèse de doctorat en criminologie, Montréal, Université de Montréal, $291 \mathrm{p}$.

Bénédicte, Manier. 1999. " Le travail des enfants dans le monde ». Paris : Editions Découvertes et Syris. Collections Repères, 119 p.

Boserup, Ester. 1970. « Woman's role in economic development ». London, Gallen// Unwin : PUF, $283 \mathrm{p}$.

Bureau International du Travail 1996. Le travail des enfants au Sénégal. Dakar : 149 p.

Cissé-Niang, Aminata. 2002. "L'interdiction internationale du travail des enfants vue d'Afrique " de Regards croisés sur le droit social sous la dir. Supio, A. MSH Guépin .

Coquery-Vidrovith, Cathérine, Héléne d'Almeida Tapor, Odile Goerg et Françoise Guitart (sous la dir. de). 1992. « Les jeunes en Afrique : Évolution et rôle XIXXXe siécle (Tome 1) ». Éditions L'Harmattan.

Cordonnier, Rita. 1982. «Femmes africaines et commerce : les revendeuses de tissu de la ville de Lomé (Togo) ». Paris : ORSTOM, 190 p.

Coté, Michelle-Marguerite. 1991. Les jeunes de la rue, Montréal : Liber, 180 p.

D'Almeidia, Hélene, Topor Cathérine Coquery-Vidrovitch et Françoise Guilart (sous la dir. de ). 1992. "Les jeunes en Afrique ». Paris : l'Harmattan, 571 p.

Daune-Richard, Marie, Anne, et Devreux, Anne-Marie. 1992. « Rapports sociaux de sexes et conceptualisation sociologique». Femmes au travail, vol. 5, no 2.

Delaunay, Valérie.1996. « Le travail des fillettes en Afrique sub-saharienne. Conditions de vie et de travail des jeunes migrantes Sérére à Dakar. ". L'enfant exploité, oppression, mise au travail, prolétarisation, sous la dir. de Schlemmer 
Bernard, Paris : Karthala, ORSTOM.

Diagne, Souleymane-Bachir. 1990. «Quelques considérations sur la crise de la jeunesse». Guestu, recherche marxiste, vol. 5-6.

Diallo-Co Trung, Marina. 1992. « La crise scolaire au Sénégal, crise de l'aurorité ? ». Les jeunes en Afrique : Évolution et rôle XIX-XXe siécles (Tome 1), sous la dir. de

Cathérine Coquery-Vidrovith, Hélène d'Almeida Tapor, Odile Goerg et Françoise Guitart, p. 407-439. L'Harmattan.

Diop, Abdoulaye-Bara.1981. «La societé wolof : tradition et changement, les systèmes d'inégalité et de domination ». Paris : Karthala, 355 p.

Diop, Momar Coumba et Ouseynou Faye. 1997. Jeunes, culture de la rue et violence urbaine en Afrique, sous la dir. de Georg Hérault et Puis Adesanmi, p. 5-7.

Diop, Nafissatou. 1989. « La fécondité des adolescentes au Sénégal ». Thèse de doctorat en démographie, Montréal, Université de Montréal.

Dubar, Claude. 2000. « La socialisation construction des identités sociales et professionnelles ». Paris : Armand Colin, 225 p.

Éclaireures et Éclaireurs du Sénégal. 2000. Étude documentaire sur la pauvreté: Situation des jeunes et adolescentes au Sénégal. Dakar.

Ela, Jean Marc. 1998. «Innovations sociales et renaissance de l'Afrique noire : les défis du monde « d'en-bas» ». Paris-Montréal : L'Harmattan, 425 p.

Enda Tiers-Monde. 1993. Le travail des enfants au sénégal. Cas des «domestiques». Dakar : Enda-Editions.

.1995. Enfants en recherche et en action: une alternative africaine d'animation urbaine, Dakar : Enda-Edition.

Evina, Akam. 1998. Vie féconde des adolescentes en mileu urbain camerounais. Cameroune : IFORD.

Fall, Abdou Salam, sous la dir. de. 2002. Enquête sur la perception de la pauvreté au Sénégal. Rapport de la région de Dakar. Dakar : MEFP-PNUD, Rapport provisoire.

Fassin, Didier. 1996. «Exclusion, underclass, marginalidad ». Revue française de sociologie, vol. XXXVIII, no, p. 37-75.

Galland, Olivier. 1991. Sociologie de la jeunesse : L'entrée dans la vie adulte. Paris : Armand Collin.

Gauthier, Madeleine. 1987. « Les nouveaux visages de la pauvreté ». Montréal: 258p.

Goffman, Erving. 1989 (1963). « Stigmate ». Paris : Éditions de Minuit, 175 p.

Hurtubise, Roch, Michèle Vatz Laaroussi et Dubuc Stéphane. 2000. Jeunes de la rue et famille: des productions sociales et des stratégies collectives au travers des mouvances du réseau. Sherbrooke : Université de Sherbrooke, 214 p.

Invernizzi, Antonella. 2001. «La vie quotidienne des enfants travailleurs : Stratégies de survie et socialisation dans les rues de Lima ». Paris : L'harmattan, 286 p.

Ki-Zerbo, Joseph. 1980. « Le monde du travail et la protection de l'enfance ». : Travail et société, vol. 5 , no 4 . 
Laberge, Danielle (Sous la dir. de). 2000. «L'errance urbaine ». Montréal : MultiMondes, 439 p.

Lange, Marie-France (Sous la dir. de).1998. «L'école et les filles en Afrique, scolarisation sous conditions ». Paris : Karthala, 254 p.

Lecarme-Frassy, Mireille. 2000. «Marchandes dakaroises entre maison et marché : approche anthropologique ». Paris, Montréal : L'Harmattan, Collections Études africaines, $267 \mathrm{p}$.

Locoh, Thèrese-Lauras et Nuria Escartin-Lopez. 1992. " Jeunesse et démographie en Afrique ». Les jeunes en Afrique : Évolution et rôle $X I X-X X$ e siécle (Tome 1), sous la dir. de Catherine Coquery-Vidrovith, Hélène-d'Almeida Tapor, Odile Georg et Françoise Guitart, p. 66-79. Paris : L'Harmattan.

Lucchini, Riccardo. 1996. «Sociologie de la survie : l'enfant dans la rue ». Paris : Presses Universitaires de France (PUF), 315 p.

Ly, Boubacar. 1979. « La situation de l'enfant dans le monde africain ». Aspects de l'éducation de l'enfant africain, UNESCO, p. 9-27.

Marcoux, Richard. 1993. «L'école ou le travail. L'activité des enfants et les caractéristiques des ménages en milieu urbain au Mali ». Thèse de doctorat en démographie,

Montréal, Université de Montreàl, 268 p.

Mendelievich, Ellias (Sous la dir. de).1980. «Le travail des enfants ». Genève : Bureau international du travail, $175 \mathrm{p}$.

Nations-Unies. 1990. Déclaration mondiale en faveur de la survie, de la protection et du développement de l'enfant et Plan d'action pour l'application de la déclaration mondiale en faveur de la survie, de la protection et du développement de l'enfant dans les années 1990. New-York : Nations Unies, 24 p.

Ndione, Emmanuel, Maximilien Sagna et Jacques Bugnicourt. 1987. « Pauvreté ambiguë : enfants et jeunes au Sénégal ». Dakar : 182 p.

Oakley, Ann. 1978 (revised edition 1985). Sex, Gender and Society: Quinze ans d'bistoire de femmes. Gower.

République du Sénégal. 1991. Plan d'action national pour l'Enfant. Dakar. 2002. Document de stratégie de réduction de la pauvreté. Dakar.

République du Sénégal, Ministère de la Femme de l'enfant et de la famille. Juillet, 1993. Femmes sénégalaises à l'horizon 2015.

République du Sénégal et commune de Dakar. 1990. Études préliminaires, réhabilitation des marchés (Kermel, Sandaga, Tiléne). Dakar.

Rodgers, Gerry and Standing Guy. 1981. «Le rôle économique des enfants dans les pays à faibles revenus». Revue International du travail, vol. 120, no 1.

Roulleau-Berger, Laurence et Nicole Chantale. 1980-2000. Les jeunes et le travail. Paris : PUF.

Sadio, Abdoulaye. 1993. Le travail des enfants au Sénégal : enquête méthodologique. Dakar : Unicef. 
Schlemmer, Bernard (Sous la dir. de ). 1996. «L'enfant exploité, Oppression, mise au travail, prolétarisation ». Karthala ORSTOM, 7-51 p.

Sow, Fatou. 1991. Les initiatives féminines au Sénégal, une réponse à la crise? (IFANCAD)

Unicef et Enda Tiers-Monde. 1996. Les Mbindaan sans Mbindou, les petites bonnes à Dakar. Dakar : Bureau international du travail.

Unicef, IFAN et IRD. 2002. Enquête auprès des adolescentes dans les régions de Dakar et Thiès

Young,Lorraine.2003. "The place of street children in Kampala,Uganda:marginalisation, resistance and acceptance in the urban environment ». Environment and Planning D. 